\title{
Effect of Different Antibiotic
} Chemotherapies on Pseudomonas aeruginosa Infection In Vitro of Primary Human Corneal Fibroblast Cells

\author{
Maria del Mar Cendra ${ }^{1}$, Myron Christodoulides ${ }^{1 *+}$ and Parwez Hossain ${ }^{2 \dagger}$ \\ ${ }^{1}$ Molecular Microbiology, Academic Unit of Clinical and Experimental Sciences, Faculty of Medicine, University of \\ Southampton, Southampton, United Kingdom, ${ }^{2}$ Eye Unit, Academic Unit of Clinical and Experimental Sciences, University \\ Hospital Southampton NHS Foundation Trust, Southampton General Hospital, Southampton, United Kingdom
}

\section{OPEN ACCESS}

Edited by:

Yuji Morita

Aichi Gakuin University, Japan

Reviewed by:

Suzanne Mariane Janete Fleiszig, University of California, Berkeley,

United States

Françoise Van Bambeke, Université catholique de Louvain,

Belgium

Jaya Devi Chidambaram, London School of Hygiene

and Tropical Medicine, United Kingdom

*Correspondence:

Myron Christodoulides m.christodoulides@soton.ac.uk

${ }^{\dagger}$ These authors have contributed equally to this work as principal investigators.

Specialty section:

This article was submitted to Antimicrobials, Resistance

and Chemotherapy,

a section of the journal

Frontiers in Microbiology

Received: 17 May 2017 Accepted: 08 August 2017

Published: 22 August 2017

Citation:

del Mar Cendra M,

Christodoulides $M$ and Hossain $P$ (2017) Effect of Different Antibiotic Chemotherapies on Pseudomonas aeruginosa Infection In Vitro of Primary Human Corneal Fibroblast

Cells. Front. Microbiol. 8:1614. doi: 10.3389/fmicb.2017.01614
Pseudomonas aeruginosa is a major cause of bacterial keratitis (BK) worldwide. Inappropriate or non-optimal antibiotic chemotherapy can lead to corneal perforation and rapid sight loss. In this study, we tested the hypothesis that $P$. aeruginosa strain PAO1 invades primary human corneal fibroblasts (hCFs) in vitro and persists intracellularly, despite chemotherapy with antibiotics used commonly to treat BK. In rank order, ciprofloxacin, levofloxacin and polymyxin B showed the highest activity against planktonic PAO1 growth (100\% inhibitory concentration $\leq 10 \mu \mathrm{g} / \mathrm{mL} ; 50 \%$ inhibitory concentration $\leq 1 \mu \mathrm{g} / \mathrm{mL}$ ), followed by gentamicin and ofloxacin (100\% inhibitory concentration $\leq 50 \mu \mathrm{g} / \mathrm{mL} ; 50 \%$ inhibitory concentration $\leq 10 \mu \mathrm{g} / \mathrm{mL}$ ). These bactericidal antibiotics (50-200 $\mu \mathrm{g} / \mathrm{mL}$ concentrations) all killed PAO1 in the extracellular environment of infected hCF monolayers. By contrast, the bactericidal antibiotic cefuroxime and the bacteriostatic antibiotic chloramphenicol failed to sterilize both PAO1 broth cultures, even at a concentration of $\geq 200 \mu \mathrm{g} / \mathrm{mL}$ ) and infected hCF monolayers. Statistically, all antibiotics were able to prevent $\mathrm{LDH}$ release from PAO1infected hCF monolayers at both concentrations tested. Intracellular Pseudomonas were significantly reduced (>99\%, $P<0.05$ ) following treatment with ciprofloxacin, levofloxacin and ofloxacin, whereas gentamicin, polymyxin B and cefuroxime failed to clear intracellular bacteria over $24 \mathrm{~h}$. Intracellular Pseudomonas infection was resistant to chloramphenicol, with hCF death observed by $9 \mathrm{~h}$. Eventual growth of remaining intracellular Pseudomonas was observed in hCF after removal of all antibiotics, resulting in re-infection cycles and cell death by $48 \mathrm{~h}$. All of the antibiotics reduced significantly $(P<0.05) \mathrm{IL}-1 \beta$ secretion by hCF infected with a Multiplicity Of Infection $(\mathrm{MOI})=1$ of PAO1. With higher $\mathrm{MOI}$, no pro-inflammatory effects were observed with antibiotic treatment, expect with polymyxin $B$ and ofloxacin, which induced significant increased IL-1 $\beta$ secretion $(P<0.001)$. The findings from our study demonstrated that bactericidal and bacteriostatic antibiotics, routinely used to treat $\mathrm{BK}$, failed to eradicate Pseudomonas infection of hCFs in vitro and that their bactericidal efficacies were influenced by the cellular location of the organism.

Keywords: Pseudomonas aeruginosa, bacterial keratitis, antibiotic chemotherapy, intracellular persistence, corneal diseases, in vitro model 


\section{INTRODUCTION}

Pseudomonas aeruginosa is the leading cause of bacterial keratitis (BK) associated with contact lens wear (Shah et al., 2011; Stapleton and Carnt, 2012). Like other Pseudomonas infections, untreated or inappropriately treated $\mathrm{BK}$ can lead to rapid progression with adverse outcomes from rapid and permanent sight loss from corneal opacification/perforation (Donzis, 1998; Sy et al., 2012; Vazirani et al., 2015). A healthy cornea is inherently resistant to microbial infections but when the epithelium is breached, e.g., following surgery, trauma or contact lens wear, microbes can penetrate and gain access to the stroma (Taube et al., 2015). Infection can become established in the corneal stroma and is characterized by bacterial growth and dissemination (Badenoch and Coster, 1989; O’Brien, 2003; Ong and Corbett, 2015). In response to bacteria-induced injury and inflammation, resident stromal keratocytes can transform into corneal fibroblasts and exacerbate inflammation by secreting proinflammatory mediators and recruiting leukocytes (Smith et al., 1997; Wong et al., 2011).

Treatment of Pseudomonas keratitis requires disinfection of the eye with effective antibiotics. Some pathogens, including Pseudomonas spp., can adapt their lifestyle to avoid innate immune defenses and antimicrobial activities, e.g., by penetrating inside eukaryotic host cells or by forming biofilms (Carryn et al., 2003; del Pozo and Patel, 2007; Lebeaux et al., 2014; Rybtke et al., 2015). Cellular localization of the bacteria and differences in the pharmacodynamic properties of antibiotics used routinely, may have a high impact on the treatment and successful chemotherapy can be difficult if the required inhibitory antibiotic dosages are not achieved (Carryn et al., 2003). Failure to achieve bacteriological eradication may be a factor in the persistence of Pseudomonas infection within the tissue as a result of the invasive genotype (Shen et al., 2015) and recurrent infection despite antibiotic treatment (Ti et al., 2007). Additionally, overstimulation of the inflammatory response is a common issue during BK treatment (Taube et al., 2015). It is not known also if different antibiotic regimens have inherent inflammatory effects that may impact on the corneal healing response. Corticosteroids are known to possess anti-inflammatory properties, but their use is not recommended until infection is cleared (Gokhale, 2008).

A large literature reports animal models for investigating bacterial factors and host cellular responses in BK (Marquart, 2011). P. aeruginosa has been reported to invade corneal epithelial cells of the mouse and rabbit in vivo (Fleiszig et al., 1996; Lee et al., 2003a; Yamamoto et al., 2006). In these studies, an inverse correlation was observed between cytotoxicity and bacterial invasion (Fleiszig et al., 1996). Furthermore, the invasive or cytotoxic background of the $P$. aeruginosa strain appeared to affect keratitis treatment of murine models in vivo (Lee et al., 2003b). The aminoglycoside antibiotic tobramycin, which poorly penetrates eukaryotic cell membranes, was found to be less effective at eradicating invasive $P$. aeruginosa strains in mouse corneas, than the penetrating fluoroquinolone antibiotic ofloxacin (Lee et al., 2003 b). In humans, an increase in the number of isolates resistant to fluoroquinolones was observed if $P$. aeruginosa encoded the type three secreted cytotoxin gene exoU (Borkar et al., 2014). Patients with ulcers caused by fluoroquinoloneresistant $P$. aeruginosa that expressed exoU trended toward poorer visual outcomes than patents with ulcers caused by bacteria that were non-resistant but still expressed exoU.

A very large literature exists also on the ability of $P$. aeruginosa to adhere to cultured myeloid and non-myeloid, primary and transformed mammalian cells in vitro and in vivo, and to subsequently invade (Bucior et al., 2014). For example, $P$. aeruginosa can adhere to and invade non- phagocytic cells such as human A549 lung epithelial cells and Caco-2 colon cells, monocytic THP-1 cells, and also different human primary cells, e.g., epithelial cells from the cornea or nose (Fleiszig et al., 1995; Esen et al., 2001; Ulrich et al., 2005; Kim and Wei, 2007; Ahmed et al., 2014). In addition, the key mechanisms of adherence and invasion, as well as intracellular persistence, pathogen exit and avoidance of innate immunity have been identified along with several of the Pseudomonas and host cellular and genetic factors involved (Bleves et al., 2010; Gellatly and Hancock, 2013; Lovewell et al., 2014). The bactericidal activity of different antibiotics against extracellular and intracellular Pseudomonas growth also has been investigated in some of these in vitro cell culture models (Smith et al., 2000; Ulrich et al., 2005; Kim and Wei, 2007; Buyck et al., 2013). Recently, we reported that $P$. aeruginosa can adhere to human primary corneal fibroblasts (hCFs) in vitro and subsequently invade. We also reported that hCFs were a source of IL- $1 \beta$ secretion in response to challenge with $P$. aeruginosa in vitro (Cendra et al., 2017). IL-1 $\beta$ is a pleiotropic cytokine that is involved in several inflammatory processes involving a variety of different eukaryotic cells (Ren and Torres, 2009) and over-stimulated IL-1 $\beta$ expression can contribute to impaired healing (Okamoto et al., 2004; Stapleton et al., 2008). In the current study, we tested the hypothesis that $P$. aeruginosa strain PAO1 invading primary human corneal fibroblasts (hCFs) in vitro could persist intracellularly, despite chemotherapy with antibiotics used commonly to treat $\mathrm{BK}$, e.g., fluoroquinolone (ciprofloxacin, levofloxacin, ofloxacin), aminoglycoside (gentamicin), polypeptide (polymyxin B), semisynthetic cephalosporin (cefuroxime) and synthetic bacteriostatic (chloramphenicol) antibiotics. We have screened and compared the pharmacodynamics, antimicrobial and antiinflammatory activities of these different antibiotic therapies currently available for clinical use in the United Kingdom to treat BK (Tuft and Burton, 2013), and ranked them according to their impact on Pseudomonas infection of hCFs in vitro and on Pseudomonas intracellular persistence.

\section{MATERIALS AND METHODS}

\section{Bacteria and Growth Conditions}

Pseudomonas aeruginosa strain PAO1 (Holloway1C Stanier131) was obtained from the National Collection of Industrial, Food and Marine Bacteria (NCIMB), United Kingdom. PAO1 was 
grown in Luria-Bertani (LB, Oxoid) broth and on nutrient agar (Oxoid) at $37^{\circ} \mathrm{C}$.

\section{Primary Human Corneal Fibroblast Cells}

Corneal epithelium was scraped from corneo-scleral rims of healthy donors and the stromal layer was dissected and digested with collagenase type-1 ( $1 \mathrm{mg} / \mathrm{mL}$; Life Technologies) for $3 \mathrm{~h}$ at $37^{\circ} \mathrm{C}$. Digested stroma was cultured in Dulbecco's Modified Eagle's Medium (DMEM, Sigma-Aldrich) supplemented with $5 \%$ (v/v) decomplemented Fetal Calf Serum (dFCS, Life Technologies), 100 Units/mL of penicillin and $100 \mathrm{Units} / \mathrm{mL}$ of streptomycin (Life Technologies) and $0.5 \mu \mathrm{g} / \mathrm{mL}$ of amphotericin B (Lonza) and fibroblasts were characterized as described previously (Wong et al., 2011). The number of cell passages used in this study was kept between 3 and 6 to minimize fibroblast differentiation to myofibroblast, as seen in other studies (Masur et al., 1995; Bargagna-Mohan et al., 2015). Fibroblasts from each donor were kept separate (not pooled) and used for independent experiments. Cells were incubated in a humidified atmosphere containing $5 \%(\mathrm{v} / \mathrm{v}) \mathrm{CO}_{2}$ at $37^{\circ} \mathrm{C}$.

In experiments to examine the efficacy of different antibiotics against extracellular and intracellular PAO1, hCFs were cultured in antibiotic-free DMEM supplemented with 5\% (v/v) dFCS for at least one passage prior to bacterial challenge, in order to ensure the absence of possible traces of antibiotics.

\section{Ethics Statement}

Patients provided written informed consent in accordance with the Declaration of Helsinki to use surplus corneal tissue specimens for research via the NHS Blood Transplant Eye Retrieval Service. Protocols were approved by the NRES Committee South Central - Berkshire 06/Q1602/56.

\section{Antibiotics and Determination of Activity against $P$. aeruginosa Using a Broth Dilution Assay}

The antibiotics chosen for this in vitro study were based on those commonly used in United Kingdom clinical practice, namely the bactericidal antibiotics ciprofloxacin (CIP, SigmaAldrich), cefuroxime $0.5 \%$ (w/v) (CXM, Hampshire Hospitals NHS), polymyxin B (PMB, Sigma-Aldrich), gentamicin (GEN, Sigma-Aldrich), ofloxacin (0.3\% w/v) (OFX, Allergan), levofloxacin $(0.5 \% \mathrm{w} / \mathrm{v})$ (LVX, Santen) and the bacteriostatic antibiotic chloramphenicol $0.5 \%$ (w/v) (CHL, Bausch and Lomb). A broth dilution assay was done to determine PAO1 susceptibility to each antibiotic. Various concentrations of antibiotic $(0.01,0.1,1,10,50,100$, and $200 \mu \mathrm{g} / \mathrm{mL})$ were added to equal volumes of $\mathrm{LB}$ broth medium with various concentrations of bacteria $\left(\sim 10^{5}, \sim 10^{6}\right.$ and $\sim 10^{7}$ Colony Forming Units $(\mathrm{CFU}) / \mathrm{mL}$ ). Absorbance (Optical Density (OD) $\lambda_{600} \mathrm{~nm}$ ) as a measure of bacterial growth was assessed hourly up to $9 \mathrm{~h}$ and then at $24 \mathrm{~h}$ of incubation at $37^{\circ} \mathrm{C}$ with shaking $(200 \mathrm{rpm})$. Comparison of the $24 \mathrm{~h}$ OD readings for bacterial growth with and without antibiotics was used to calculate the lowest concentration of antibiotic that completely reduced OD growth by $100 \%$ and the concentration of antibiotic that reduced OD growth by $50 \%$.

\section{$P$. aeruginosa Infection of hCFs}

Extracellular and intracellular PAO1 bacteria were quantified in the presence of each antibiotic used at concentrations of 50 and $200 \mu \mathrm{g} / \mathrm{mL}$. Confluent hCF monolayers were challenged in triplicate in 24-well cell culture plates (Greiner Bio-one, $\sim 10^{5} \mathrm{hCF} /$ well) with different Multiplicities Of Infection $\left(\mathrm{MOI}=1, \sim 10^{5} \mathrm{CFU} / \mathrm{ml} ; \mathrm{MOI}=10, \sim 10^{6} \mathrm{CFU} / \mathrm{mL}\right.$ and MOI $=100, \sim 10^{7} \mathrm{CFU} / \mathrm{mL}$ ) of PAO1. At given time-points, cell monolayers were washed gently four times with phosphate buffered saline (PBS), pH7.4 and associated PAO1 bacteria were quantified by viable counting on nutrient agar after hCF lysis with a buffer of PBS containing $0.1 \%(\mathrm{w} / \mathrm{v})$ saponin and $0.1 \%(\mathrm{v} / \mathrm{v}) \mathrm{dFCS}$, as described previously (Hung et al., 2013).

The gentamicin exclusion assay was used to eliminate extracellular bacteria in order to subsequently quantify intracellular bacteria. The assay was done as described previously (Hung et al., 2013), whereby infected cell monolayers are washed with PBS, pH 7.4 and then incubated with a solution of gentamicin $(200 \mu \mathrm{g} / \mathrm{mL})$ for $90 \mathrm{~min}$ to kill extracellular bacteria. The monolayers were washed to remove gentamicin and then lysed with a solution of PBS containing saponin $(0.1 \% \mathrm{w} / \mathrm{v})$ and $\operatorname{dFCS}(0.1 \% \mathrm{v} / \mathrm{v})$ and the surviving intracellular bacteria enumerated by viable counting on NA.

To quantify the effects of the different antibiotics on survival of intracellular PAO1 bacteria, $1 \mathrm{~mL}$ of DMEM containing 50 or $200 \mu \mathrm{g} / \mathrm{mL}$ of the antibiotics listed above was added to hCFs after $3 \mathrm{~h}$ of PAOl infection at the different MOIs, and intracellular PAO1 enumerated after $1.5,4.5,7.5$, and $24 \mathrm{~h}$ of each antibiotic treatment. At these given time points, monolayers were washed four times with PBS, lysed and intracellular bacteria quantified as described above. Extracellular growth of PAO1 during cell monolayer infection with different antibiotic treatments was quantified by viable counting at $0,1,3,6,9$, and $24 \mathrm{~h}$ on nutrient agar.

\section{Quantification of Extracellular IL-1 $\beta$ Cytokine}

hCF monolayers were infected for $3 \mathrm{~h}$ with PAO1 $(\mathrm{MOI}=1,10$, and 100), then treated with antibiotics and IL-1 $\beta$ cytokine was quantified from $9 \mathrm{~h}$ antibiotic-treated-hCF supernatants using the Meso Scale Discovery (MSD) electro-chemiluminescence assay (MesoScaleDiagnostics), following the manufacturer's instructions.

\section{Cytotoxicity Activity}

The cytotoxicity of CIP, CXM, PMB, GEN, OFX, LVX and CHL (50 and $200 \mu \mathrm{g} / \mathrm{mL}$ ) alone to hCFs and of PAO1 infection (MOI $=1,10$, and 100) with and without antibiotics, was examined after $0,1,3,6$, 9, and $24 \mathrm{~h}$ of incubation. Release of lactate dehydrogenase $(\mathrm{LDH})$ was measured using the Pierce LDH Cytotoxicity 
Assay Kit (Thermo-Scientific) and the percentage cytotoxicity was calculated following the manufacturers' instructions.

\section{Statistics}

Data were analyzed by one-way ANOVA with Dunnett's multiple comparison test or by unpaired $t$-test. $P$-values $<0.05$ denoted significance.

\section{RESULTS}

\section{Pharmacodynamics of Antibiotic Chemotherapies for $P$. aeruginosa PAO1 Infection of Primary hCF Cultures In Vitro}

In the current study, we used an in vitro model of primary hCF cells derived from the stroma, to evaluate current antibiotic treatments used in the United Kingdom to treat eye infections. Initially, we used a broth dilution method to calculate the lowest concentration of GEN, CXM, OFX, PMB, CIP, CHL and LVX antibiotics that completely reduced OD growth of $P$. aeruginosa strain $\mathrm{PAO} 1$ (tested at various concentrations of $\sim 10^{5}-\sim 10^{7} \mathrm{CFU} / \mathrm{mL}$ ) by values of 50 and $100 \%$. In rank order, the fluoroquinolones CIP and LVX and polymyxin PMB showed the highest antimicrobial activities with $100 \%$ reduction in $\mathrm{OD}$ with a concentration $\leq 10 \mu \mathrm{g} / \mathrm{mL}$ and $50 \%$ reduction in $\mathrm{OD}$ with a concentration $\leq 1 \mu \mathrm{g} / \mathrm{mL}$ (Table 1). This was followed by GEN and OFX (100\% reduction in OD with concentrations $\leq 50 \mu \mathrm{g} / \mathrm{mL}$ and $50 \%$ reduction in OD with concentrations $\leq 10 \mu \mathrm{g} / \mathrm{mL}$ ) and lastly by CXM and CHL treatments, which showed the lowest activities $(\geq 200 \mu \mathrm{g} / \mathrm{mL}$ ) and failed to sterilize the PAO1 broth cultures (Supplementary Figure S1 and Table S1). LB broth could support the planktonic growth of $P$. aeruginosa PAO1 over time in the absence of antibiotics (Supplementary Figure S2).

Next, we examined the bactericidal ability of the antibiotic panel to control PAO1 extracellular infection of cultured hCF cells. The antibiotic concentrations to be tested were chosen as 50 and $200 \mu \mathrm{g} / \mathrm{mL}$ based on their ability to reduce planktonic growth of PAO1 in broth (Table 1). For some of the antibiotics, e.g., CIP, LVX and PMB, these concentrations were significantly higher than the concentrations that could completely inhibit bacterial growth as judged by the broth dilution assay, but could be seen as potentially representing accumulated antibiotic concentrations in clinical use with repeated dosing of infected eye tissue. hCF cultures were infected with PAO1 $(\mathrm{MOI}=1,=10,=100)$, antibiotics added at time $=0 \mathrm{~h}$ and extracellular growth of the organism quantified (CFU) over time (Figure 1). Concentrations of 50 and $200 \mu \mathrm{g} / \mathrm{mL}$ of CIP, LVX, PMB, GEN and OFX killed all PAO1 $(>99.99 \% ; P<0.0001)$ in the extracellular environment by $1 \mathrm{~h}$ (Figures 1A-E). Treatment with CXM at $50 \mu \mathrm{g} / \mathrm{mL}$ resulted in a $>99 \%$ reduction of extracellular bacterial CFU by $6 \mathrm{~h}(P<0.05)$, but this was not maintained and bacterial CFU increased thereafter to levels similar to bacterial growth observed in PAO1-infected monolayers not

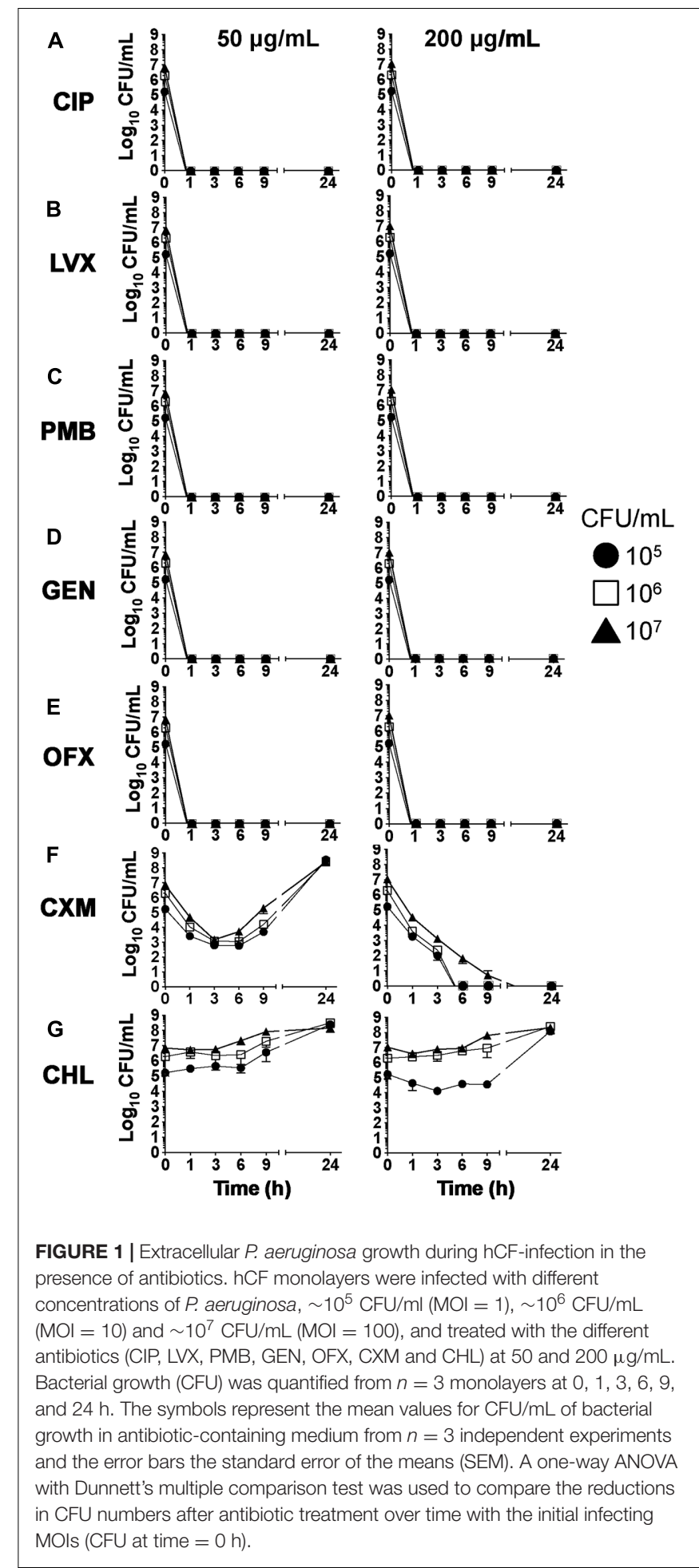

treated with antibiotics $\left(\geq 10^{8}-10^{9} \mathrm{CFU} / \mathrm{mL} ; P>0.05\right.$, using an unpaired $t$-test; Supplementary Figure S3). CXM treatment only killed extracellular PAO1 when used at a concentration of $200 \mu \mathrm{g} / \mathrm{mL}(P<0.05$ up to $6 \mathrm{~h}$ and $P<0.001$ at $24 \mathrm{~h})$, with sterility occurring between 6-9 h (Figure 1F). By contrast, $\mathrm{CHL}$ was ineffective at killing PAO1 at both concentrations 
TABLE 1 | Effect of antibiotics on the growth of $P$. aeruginosa PAO1, determined with a broth dilution assay.

\begin{tabular}{|c|c|c|c|c|c|c|c|c|c|c|c|c|c|c|}
\hline \multirow[b]{2}{*}{ PAO1 MOI } & \multicolumn{2}{|c|}{ CIP } & \multicolumn{2}{|c|}{ LVX } & \multicolumn{2}{|c|}{ PMB } & \multicolumn{2}{|c|}{ GEN } & \multicolumn{2}{|c|}{ OFX } & \multicolumn{2}{|c|}{ CXM } & \multicolumn{2}{|c|}{$\mathrm{CHL}$} \\
\hline & $100 \%$ & $50 \%$ & $100 \%$ & $50 \%$ & $100 \%$ & $50 \%$ & $100 \%$ & $50 \%$ & $100 \%$ & $50 \%$ & $100 \%$ & $50 \%$ & $100 \%$ & $50 \%$ \\
\hline 1 & $>1 ;<10$ & $>0.1 ;<1$ & 10 & $>1 ;<10$ & 10 & 1 & 10 & $>1 ;<10$ & 50 & 10 & $>200$ & 200 & $>200$ & $>200$ \\
\hline 10 & 10 & 1 & 10 & $>1 ;<10$ & 10 & 1 & 50 & 10 & 50 & 10 & $>200$ & 200 & $>200$ & $>200$ \\
\hline 100 & 10 & 1 & 10 & $>1 ;<10$ & 10 & 1 & 50 & 10 & 50 & 10 & $>200$ & 200 & $>200$ & $>200$ \\
\hline
\end{tabular}

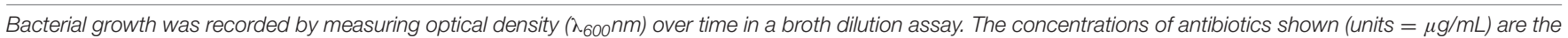
lowest concentrations that reduced optical density readings at $24 \mathrm{~h}$ completely (100\%) and by 50\%.

(Figure 1G) $(P>0.05)$, with extracellular $\mathrm{CFU}$ of $\mathrm{PAO} 1$ increasing over time to levels $\left(\geq 10^{8} \mathrm{CFU} / \mathrm{mL} ; P>0.05\right.$, using an unpaired $t$-test) similarly observed in PAO1-infected monolayers not treated with antibiotics (Supplementary Figure S3).

PAO1 infection is cytotoxic to hCFs in vitro, with MOIdependent cell death occurring between 9-24 h, as judged by loss of monolayer integrity and increased release of $\mathrm{LDH}$ due to membrane damage (Supplementary Figure S4A). We next examined whether the antibiotic treatments protected hCF monolayers from PAO1-induced cell death. In general, antibiotics alone were not cytotoxic to hCFs in vitro, except for treatment with $\mathrm{PMB}$, which increased $\mathrm{LDH}$ release by 30$40 \%$ by $24 \mathrm{~h}$, and with OFX to a lesser extent ( $\sim 10 \%$ increased LDH release) (Supplementary Figure S4B). By visual inspection, PAO1-infected hCF monolayers treated and maintained with the antibiotics CIP, LVX, GEN and PMB were intact after $24 \mathrm{~h}$. OFXtreated hCF cells showed signs of apoptosis by morphology, i.e., cell rounding and shrinkage, although the monolayers were still intact. By contrast, CXM or CHL treatments failed to protect hCF monolayers from PAO1 infection, with cell death and monolayer detachment occurring between 9 and $24 \mathrm{~h}$.

The protective effect of antibiotics was confirmed by measuring LDH release (Figure 2). Monolayers were infected with PAO1 $(\mathrm{MOI}=1,10,100)$, antibiotics were added at $3 \mathrm{~h}$ and $\mathrm{LDH}$ release measured at 9 and $24 \mathrm{~h}$. At a $\mathrm{MOI}=1$, there was no significant difference at $9 \mathrm{~h}$ in the cytotoxicity levels between CIP and GEN concentrations and no antibiotic $(P>0.05)$, whereas cytotoxicity levels were increased marginally following LVX, OFX, CXM, CHL $(P<0.05)$ and significantly with $\mathrm{PMB}(P<0.0001)$ treatments. By $24 \mathrm{~h}$, significant reductions in cytotoxicity were observed with CIP, LVX, GEN and OFX $(P<0.0001)$, PMB and CXM $(P<0.05)$ and CHL $(P<0.01)$.

At a $\mathrm{MOI}=10$, there was a significant reduction by $9 \mathrm{~h}$ in percentage cytotoxicity with 50 and $200 \mu \mathrm{g} / \mathrm{mL}$ concentrations of CIP, LVX and GEN $(P<0.001)$ and OFX $(P<0.05)$ and with $200 \mu \mathrm{g} / \mathrm{mL}$ concentration only of CXM $(P<0.05)$. With PMB treatment, there was no significant difference in cytotoxicity levels with the $50 \mu \mathrm{g} / \mathrm{mL}$ concentration $(P>0.05)$, but the $200 \mu \mathrm{g} / \mathrm{mL}$ concentration increased cytotoxicity significantly $(P<0.001)$. By $24 \mathrm{~h}$, highly significant reductions in cytotoxicity were observed with 50 and $200 \mu \mathrm{g} / \mathrm{mL}$ of CIP, LVX, PMB, GEN, OFX and CXM $(P<0.0001)$. CHL used at a concentration of $50 \mu \mathrm{g} / \mathrm{mL}$ had no significant effect on $\mathrm{LDH}$ release at either 9 or
$24 \mathrm{~h}(P>0.05)$, but percentage cytotoxicity was reduced with the higher concentration tested $(P<0.05)$ (Figure 2).

At a $\mathrm{MOI}=100$, there was a significant reduction by $9 \mathrm{~h}$ in percentage cytotoxicity with concentrations of 50 and $200 \mu \mathrm{g} / \mathrm{mL}$ of CIP and LVX $(P<0.0001)$ and GEN and OFX $(P<0.01)$ and with $\mathrm{CHL}(P<0.01,200 \mu \mathrm{g} / \mathrm{mL}$ concentration only). By contrast, $\mathrm{PMB}$ used at $200 \mu \mathrm{g} / \mathrm{mL}$ significantly increased cytotoxicity $(P<0.05)$. By $24 \mathrm{~h}$, a $50 \mu \mathrm{g} / \mathrm{mL}$ concentration of CIP, LVX, OFX, PMB, GEN and OFX significantly reduced cytotoxicity $(P<0.0001)$, but both CXM and CHL had no significant effect $(P>0.05)$. With the higher concentration of $200 \mu \mathrm{g} / \mathrm{mL}$, significant reductions were observed for all antibiotic treatments (CIP, LVX, GEN $P<0.0001$; PMB, OFX, CXM and CHL $P<0.05)$.

\section{hCF Intracellular $P$. aeruginosa Survival during Different Antibiotic Treatment}

Recently, we have shown with adherence and gentamicin exclusion assays that $P$. aeruginosa can adhere to primary hCFs and subsequently invade (Cendra et al., 2017). In the current study, MOI-dependent levels of PAO1 adherence and subsequent invasion were observed after $3 \mathrm{~h}$ of infection (Figure 3). The numbers of invasive PAO1 were quantified as $\sim 5 \times 10^{2} \mathrm{CFU} /$ monolayer with an initial MOI $=1, \sim 10^{3} \mathrm{CFU} /$ monolayer for an initial $\mathrm{MOI}=10$ and $\sim 5 \times 10^{4} \mathrm{CFU} /$ monolayer for an initial MOI $=100$ (Figure 3).

We next tested the hypothesis that the ability of PAO1 to invade hCFs enabled the organism to evade antibiotic chemotherapy, thereby creating a potential reservoir for reactivation of bacterial infection. In order to examine the effects of antibiotics on intracellular bacteria, hCF monolayers were infected with PAO1 (MOI $=1,10,100)$ for $3 \mathrm{~h}$ to allow bacterial invasion to occur (Figure 3), after which antibiotics (50 or $200 \mu \mathrm{g} / \mathrm{mL}$ ) were added to the cultures. The numbers of intracellular PAO1 were then quantified after 1.5, 4.5, 7.5, and $24 \mathrm{~h}$ of antibiotic treatment. A clear rank order for antibiotic efficacy was observed against intracellular PAO1 in vitro. The fluoroquinolones CIP, LVX and OFX demonstrated the highest antimicrobial activity (Figure 4A). Treatment with CIP and LVX (50 and $200 \mu \mathrm{g} / \mathrm{mL}$ ) killed $>99.99 \%$ of all intracellular bacteria by $3 \mathrm{~h}$, regardless of MOI used to infect the monolayers (Figures 4A,B). Treatment with OFX (50 or $200 \mu \mathrm{g} / \mathrm{mL}$ ) eradicated $>99.99 \%$ of intracellular bacteria by $3 \mathrm{~h}$ from hCF monolayers infected initially with an $\mathrm{MOI}=1$ (Figure 4C). With 


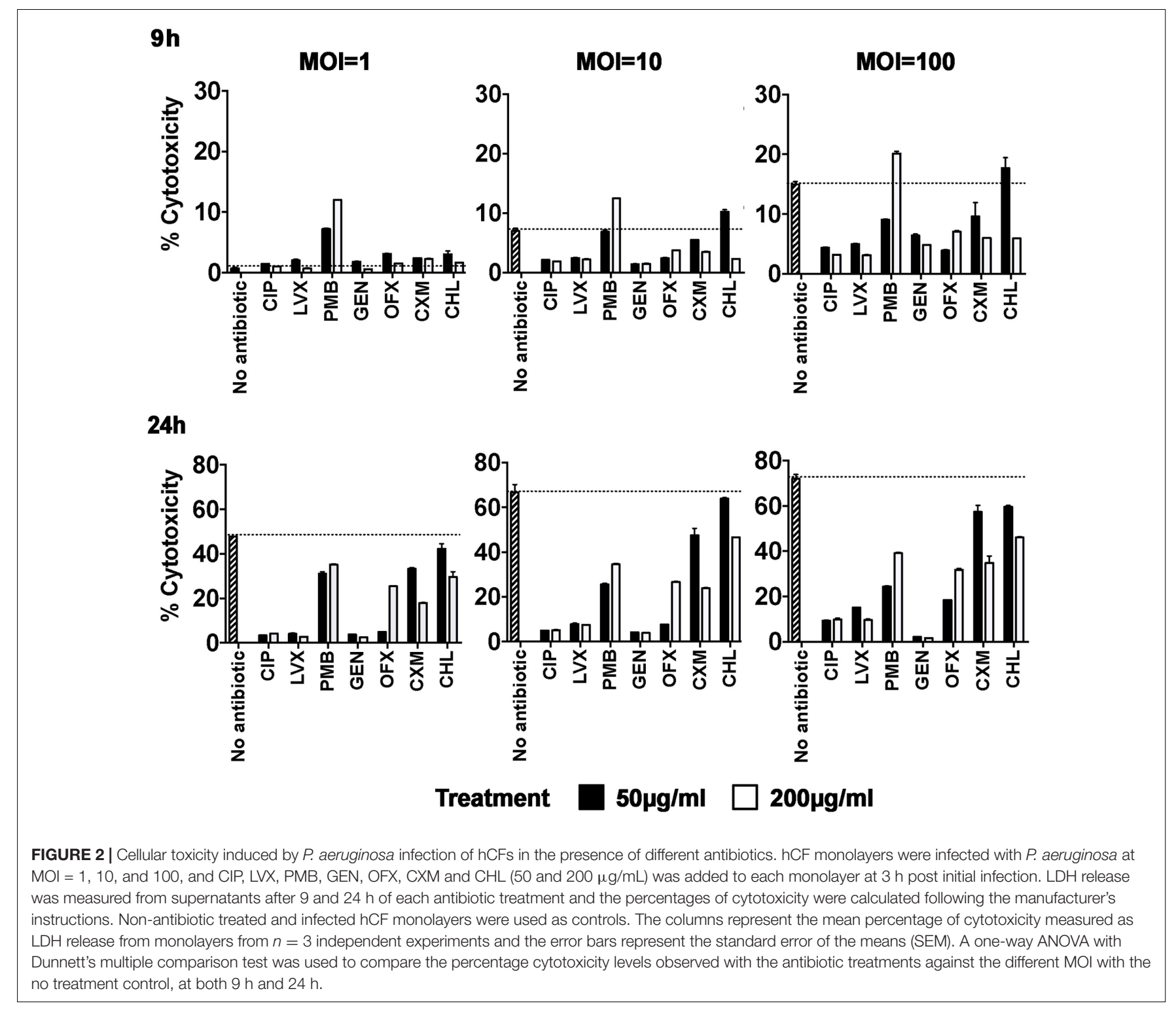

increasing MOI $=10-100$, between $\sim 10^{2}-\sim 10^{3}$ intracellular bacteria were recovered by $24 \mathrm{~h}$ after $\operatorname{OFX}(50$ or $200 \mu \mathrm{g} / \mathrm{mL}$ ) treatment, which still represented high antimicrobial activity of $>99.9 \%$ reduction in CFU (Figure 4C).

By contrast to the fluoroquinolones, treatment with GEN, $\mathrm{PMB}, \mathrm{CXM}$ or CHL failed to clear intracellular PAO1 over the $24 \mathrm{~h}$ time course of the experiments. With cells infected with MOI $=1,10$, or $100, \sim 10^{3}-\sim 10^{5}$ intracellular bacteria were recovered after treatment for $3 \mathrm{~h}$ with GEN or PMB (50 and $200 \mu \mathrm{g} / \mathrm{mL}$ ), and these intracellular PAO1 numbers essentially remained unchanged by $24 \mathrm{~h}$ (Figures 4D,E). CXM treatment at $50 \mu \mathrm{g} / \mathrm{mL}$ could not clear the infection (intracellular or extracellular), and exponential increases in bacterial CFU were observed (Figure 4F), and the numbers of intracellular PAO1 enumerated at $3 \mathrm{~h}\left(\sim 10^{3}-\sim 10^{5} \mathrm{CFU}\right)$ in the presence of $200 \mu \mathrm{g} / \mathrm{mL}$ of CXM remained essentially unchanged by $24 \mathrm{~h}$ (Figure 4F). These experiments confirmed also that PAO1 was resistant to $\mathrm{CHL}$, with exponentially increasing numbers of bacteria recovered over time intracellular and extracellularly (Figures 2, 4F and Supplementary Table S2), with cell death occurring early by $9 \mathrm{~h}$.

Clinically, a high incidence of recurrent BK has been observed in patients after cessation of antibiotic treatment (Kaye et al., 2013). We next tested the hypothesis that culture sterility and monolayer integrity were unaffected after removal of the antibiotics at $24 \mathrm{~h}$. hCFs were infected with PAO1 at a MOI $=1$ in order to detect logarithmic changes in PAO1 growth, for $3 \mathrm{~h}$ prior to the addition of antibiotics, as described above. At $24 \mathrm{~h}$, antibiotic-containing media were removed, the cell monolayers washed and fresh antibiotic-free medium added. Extracellular and intracellular PAO1 growth was then measured at time intervals up to $48 \mathrm{~h}$ after removal of antibiotics. We compared the fluoroquinolones CIP, LEV and OFX along with GEN and PMB, but excluded the CXM and CHL antibiotic treatments due 


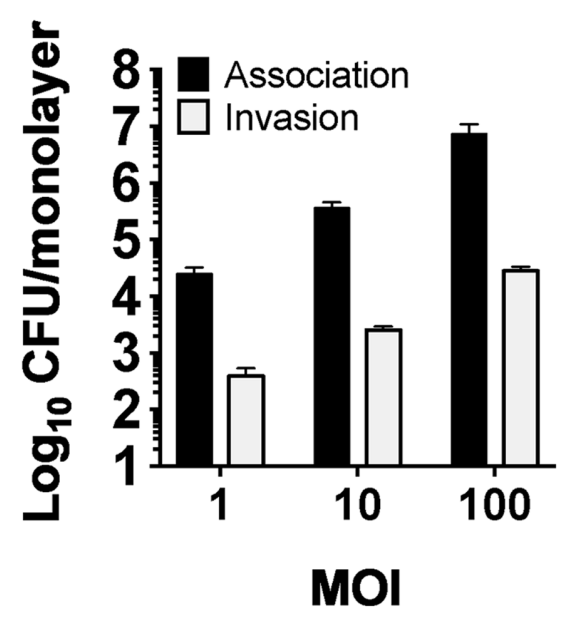

FIGURE 3 | Association of PAO1 to hCF monolayers and subsequent invasion. The graph shows the association of PAO1 to hCF-monolayers after $3 \mathrm{~h}$ of infection at $\mathrm{MOI}=1,10,100$, and the levels of invasion measured by quantifying intracellular PAO1 CFU after 90 min of GEN treatment at $200 \mu \mathrm{g} / \mathrm{mL}$ (using a standard gentamicin protection assay). The columns represent the mean $\mathrm{CFU} /$ monolayer and the error bars the standard error of the mean (SEM) of $n=3$ independent experiments.

to their inability to limit infection. Intracellular PAO1 growth was eventually detected in all monolayers after removal of antibiotics at $24 \mathrm{~h}$. With CIP and LVX-treated monolayers, intracellular PAO1 growth was detected as early as $3 \mathrm{~h}$ after antibiotic removal and increased exponentially over time to $24 \mathrm{~h}$ (Figures 5A,B). With OFX, intracellular PAO1 were recovered from infected hCF-monolayers at $24 \mathrm{~h}$ (Figure 4) and after antibiotic removal, intracellular CFU numbers showed similar exponential increases up to $24 \mathrm{~h}$ (Figure 5C). With these three treatments, extracellular bacteria were recovered by $9 \mathrm{~h}$ onward after antibiotic removal and CFU numbers increased exponentially (Figures 5A-C). For both PMB and GEN, which failed to clear intracellular bacteria after $24 \mathrm{~h}$ (Figure 4), removal of the antibiotics resulted in increased CFU numbers intracellularly and early recovery of extracellular bacteria (Figures 5D,E). Regardless, after removal at $24 \mathrm{~h}$ of all the different antibiotics, by $24-48 \mathrm{~h}$ later, all the monolayers were destroyed as a consequence of uncontrolled bacterial growth. All the bacteria recovered at 48 and 96 h now represented growth in host cell-free cultures.

\section{Effect of Antibiotic Treatment on IL-1 $\beta$ Cytokine Release by $P$. aeruginosa -Infected hCFs}

Infection of hCFs with $P$. aeruginosa PAO1 induces significant extracellular release of the cytokine IL-1 $\beta$ (Wong et al., 2011; Cendra et al., 2017). Since exacerbation of the inflammatory response is a critical event during eye infection, we tested the hypothesis that antibiotic treatments not only reduced bacterial infection but were also anti-inflammatory, by influencing IL-1 $\beta$ cytokine production. Our cytotoxicity data demonstrated that after $9 \mathrm{~h}$, untreated PAO1 infection appeared to compromise hCF monolayer integrity; therefore, IL-1 $\beta$ cytokine was measured

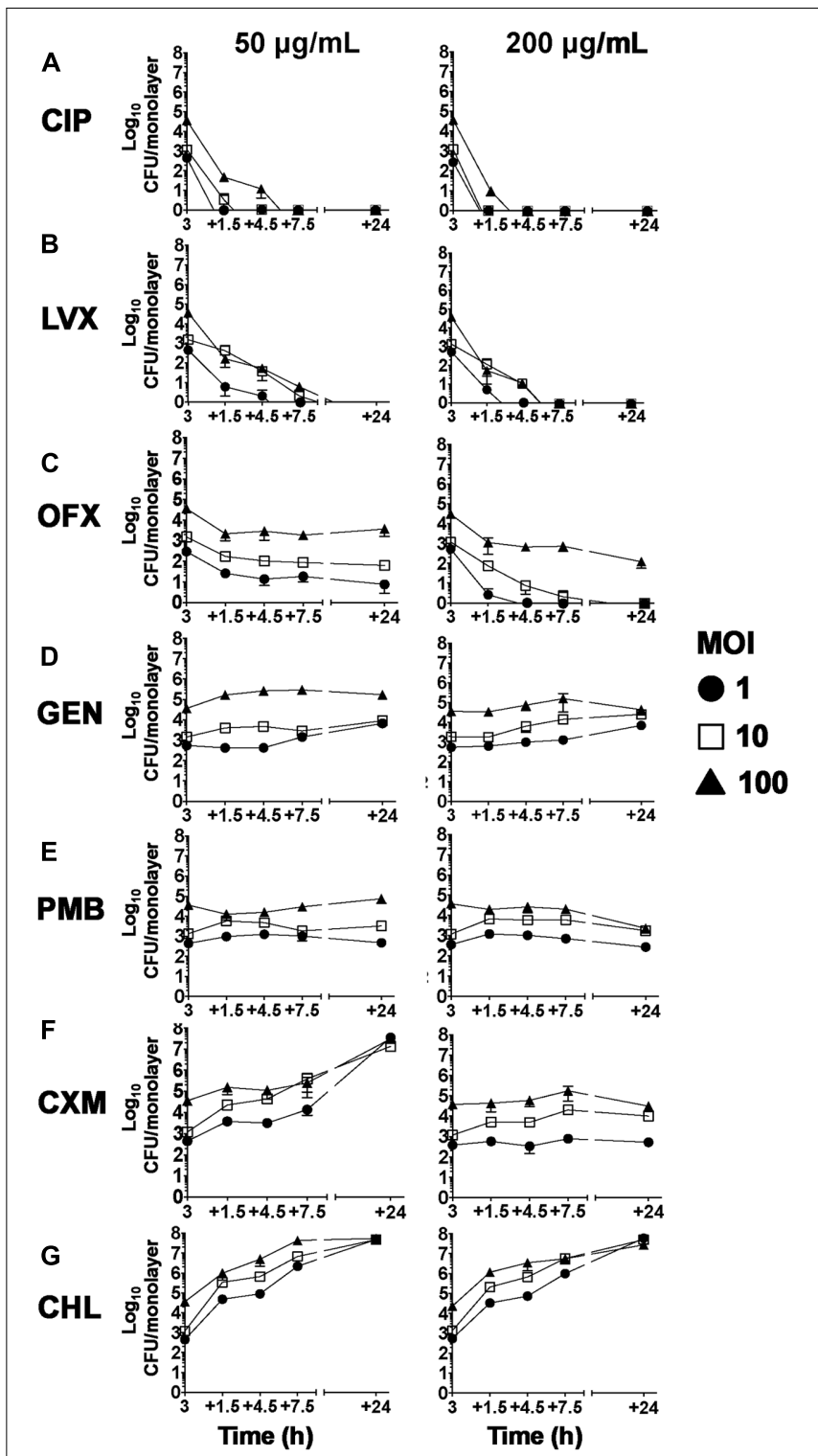

FIGURE 4 | Effect of antibiotic treatments against hCF-intracellular $P$. aeruginosa. Antibiotic treatment at 50 and $200 \mu \mathrm{g} / \mathrm{mL}$ in hCF monolayers infected with PAO1 wild-type $(\mathrm{MOI}=1,10$, and 00$)$. The symbols represent the mean and the error bars the SEM from $n=3$ independent experiments, of intracellular PAO1 CFU that was enumerated by viable counting onto agar plates after $1.5,4.5,7.5$, and $24 \mathrm{~h}$ of each antimicrobial treatment that was added $3 \mathrm{~h}$ after initial infection. With CXM and $\mathrm{CHL}$ treatment, the PAO1 CFU data represent the sum of associated and invaded bacteria. A one-way ANOVA with Dunnett's multiple comparison test was used to compare the intracellular CFU numbers at each time point after antibiotic addition (50 and $200 \mu \mathrm{g} / \mathrm{mL}$ ) with those recorded at $3 \mathrm{~h}$ before addition of antibiotics and for each $\mathrm{MOI}$ used. All calculated $P$-values are shown in Supplementary Table S2.

at this time-point in supernatants of PAO1-infected $(\mathrm{MOI}=1$, $10,100)$ and un-infected hCFs (MOI $=0)$ monolayers following addition of the different antibiotics after $3 \mathrm{~h}$ of infection (Figure 6). Baseline levels of IL-1 $\beta$ in uninfected monolayers without antibiotic treatments were $\leq 0.25 \mathrm{pg} / \mathrm{mL}$ (Figure 6A). 


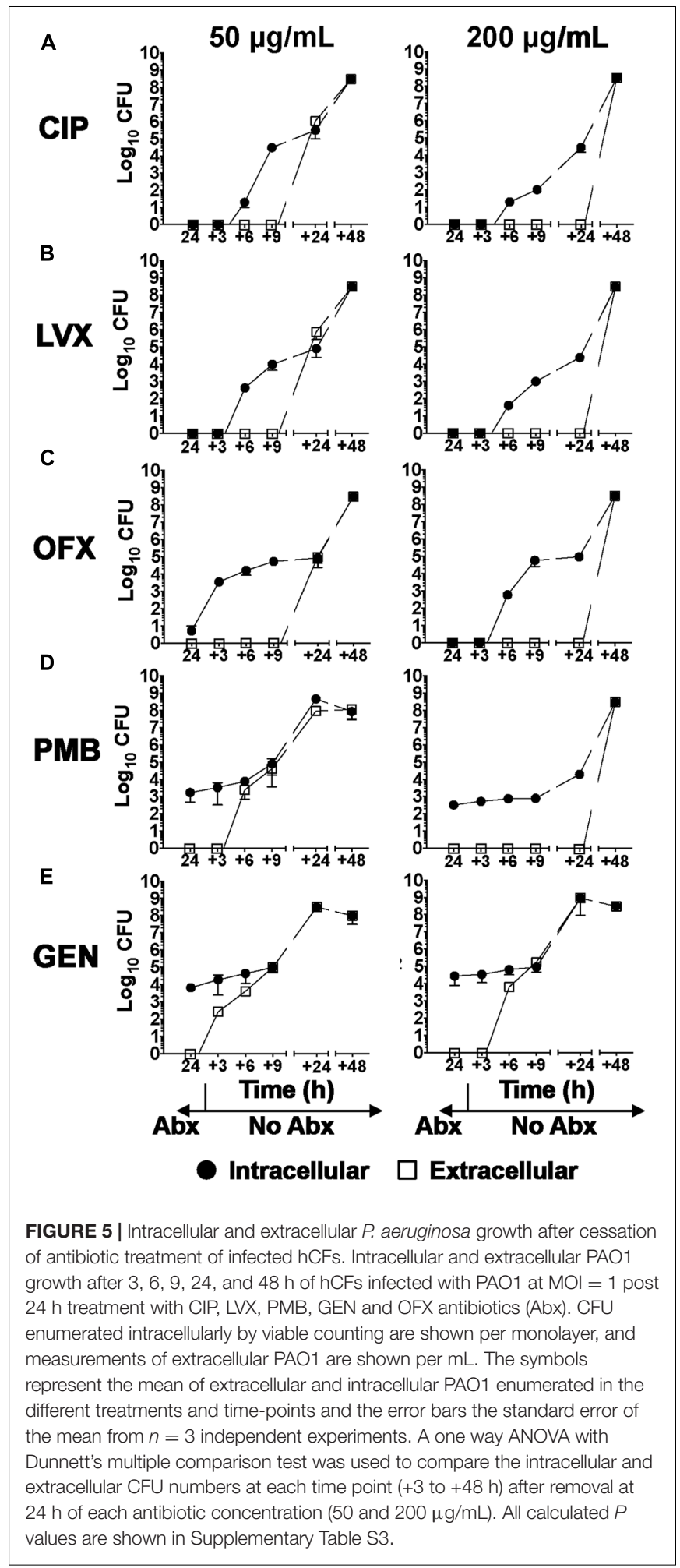

Addition of some of the antibiotics, i.e., PMB, OFX, CXM and $\mathrm{CHL}$, did statistically increase IL- $1 \beta$ release from uninfected monolayers, but the levels never exceeded $6 \mathrm{pg} / \mathrm{mL}$ (Figure 6A and Supplementary Table S4).
Increased levels of IL-1 $\beta$ production were observed with increasing PAO1 MOI: with $\mathrm{MOI}=1$, IL- $1 \beta$ levels with no antibiotic were $\sim 6 \mathrm{pg} / \mathrm{mL}$ and addition of all the antibiotics at both concentrations significantly reduced these low levels to $\leq 3$ $\mathrm{pg} / \mathrm{mL}$ (Figure 6B and Supplementary Table S4). With MOI $=10$, IL- $1 \beta$ levels with no antibiotic were $\sim 15-20 \mathrm{pg} / \mathrm{mL}$ and addition of all the antibiotics at a concentration of $50 \mu \mathrm{g} / \mathrm{mL}$ either reduced (CIP, LVX, GEN, OFX) or had no significant stimulatory effect (PMB, CXM, CHL) on IL-1 $\beta$ secretion (Figure 6C and Supplementary Table S4). Similarly, addition of $200 \mu \mathrm{g} / \mathrm{mL}$ of the antibiotics either reduced (CIP, PMB, GEN) or had no significant stimulatory effect (LVX, OFX, CXM, CHL) on IL-1 $\beta$ levels, except for PMB, which significantly increased $(P<0.0001)$ IL-1 $\beta$ levels $(\geq 40 \mathrm{pg} / \mathrm{mL}$ ) in the presence of bacteria (Figure $6 \mathrm{C})$. With MOI $=100, \sim 50-60 \mathrm{pg} / \mathrm{mL}$ IL-1 $\beta$ was induced (Figure 6D) and none of the antibiotics used at a concentration of $50 \mu \mathrm{g} / \mathrm{mL}$ statistically reduced IL- $1 \beta$ release (Supplementary Table S4). Only CIP and GEN treatment at $200 \mu \mathrm{g} / \mathrm{mL}$ significantly decreased IL$1 \beta$ release by $\geq 50 \%$ (Supplementary Table $S 4$ ). PMB, CXM and CHL had no anti-inflammatory effect with similar levels of IL-1 $\beta$ release $(P>0.05)$ measured, whereas addition of $200 \mu \mathrm{g} / \mathrm{mL}$ of OFX was pro-inflammatory, significantly $(P<0.001)$ increasing IL- $1 \beta$ release by $\sim 2$-fold $(>100 \mathrm{pg} / \mathrm{mL}$ ) compared to no antibiotic, infected hCF cells (Figure 6D and Supplementary Table S4). Notably, cells infected with MOI = 100 and treated with OFX $(200 \mu \mathrm{g} / \mathrm{mL})$ were morphologically apoptotic, which was not observed in PAO1 infected cells treated with any of the other antibiotics.

\section{DISCUSSION}

The key findings from the current study were that some bactericidal antibiotics routinely used to treat BK failed to eradicate $P$. aeruginosa infection of primary hCFs and some exacerbated the host cell cytokine inflammatory response. Bactericidal efficacy of the antibiotics was influenced by the cellular location of the organism. In clinical practice, antibiotic dosing regimens vary significantly from practice to practice, and typically in severe infections, topical antibiotic(s) are administrated hourly to the eye. However, these agents are diluted by the tears with the majority draining through the nasolacrimal duct (O'Brien, 2003). It is not known how much antibiotic penetrates into the infected tissue; in our in vitro study, we mimicked a high concentration of antibiotics commonly used to treat BK in the United Kingdom, with a caveat that maintaining high concentrations of antibiotics in essentially closed systems for long periods of time potentially over-estimates antibiotic exposure.

In the current study, we demonstrated that $P$. aeruginosa adhered to primary hCFs in vitro and subsequently invaded and replicated intracellularly. Such observations have been reported also with corneal epithelial cells (Fleiszig et al., 1995) and are common with other intracellular pathogens and host cell types (Gresham et al., 2000; Hammerschlag, 2002; Young et al., 2002; Cosma et al., 2003; Flannagan et al., 2016). It is possible 

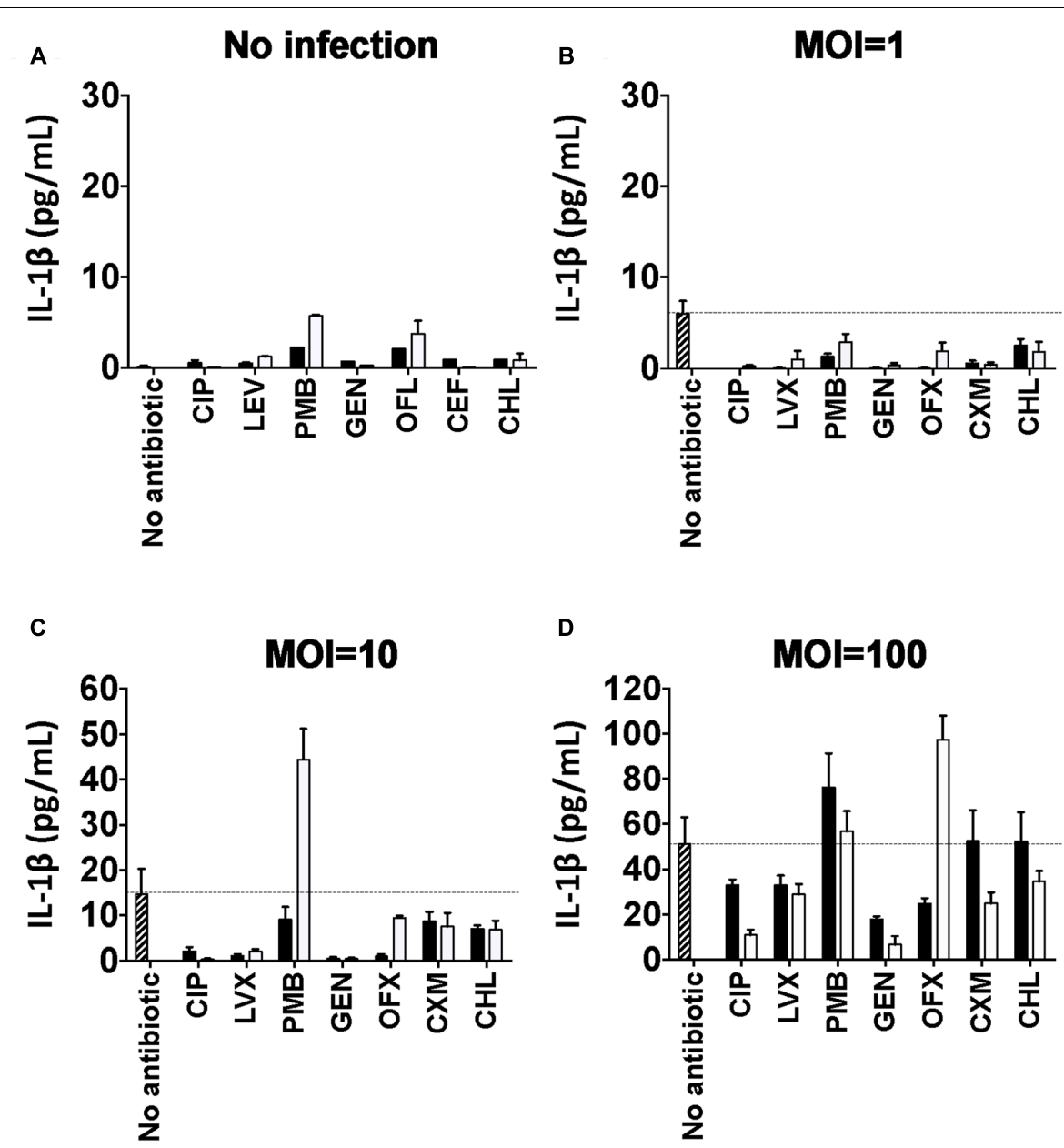

D

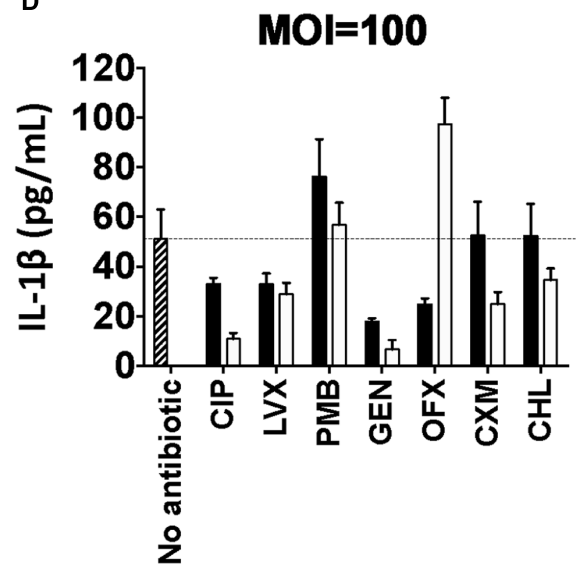

Treatment $\square 50 \mu \mathrm{g} / \mathrm{ml} \square 200 \mu \mathrm{g} / \mathrm{ml}$

FIGURE 6 | Pro-inflammatory IL-1 $\beta$ cytokine release in PAO1-infected hCFs after antibiotic treatment. MSD-ELISA quantifications of extracellular matured IL-1 $\beta$ in hCFs-supernatants after $9 \mathrm{~h}$ of No antibiotic, CIP, LVX, OFX, CXM, GEN, PMB, CHL treatment at 50 and $200 \mu \mathrm{g} / \mathrm{mL}$ of uninfected (A) and PAO1-infected monolayers at $\mathrm{MOI}=1$ (B), $=10$ (C) and $=100$ (D) for $3 \mathrm{~h}$. Maximal IL-1 $\beta$ release by hCF infected with PAO1 without antibiotic treatment is marked with a line as reference. The columns represent the mean of extracellular quantification of $\mathrm{IL}-1 \beta$ release and the error bars the standard error of the means (SEM) of $n=3$ independent experiments. A one way ANOVA with Dunnett's multiple comparison test was used to compare the levels of IL-1 $\beta$ cytokine production in the presence of antibiotic against no antibiotic, for each of the MOI conditions. All calculated $P$ values are shown in Supplementary Table S4.

that internalization of $P$. aeruginosa within corneal epithelial cells and stromal hCFs is a general mechanism employed by this organism to evade immune detection in the cornea and also indirectly to avoid antimicrobial chemotherapy, especially from antibiotics that slowly penetrate eukaryotic cells. The common features of phagocytosis and the ability of some bacteria to survive and replicate intracellularly favors pathogen mobilization within the host and suggests that prolonged antibiotic treatment is likely to be required for complete clearance of infection (Wilson et al., 2002; Cosma et al., 2003). However, P. aeruginosa, in common with many other bacterial pathogens, is likely to use other strategies to avoid the bactericidal effects of antimicrobial compounds. These include biofilm-formation, especially on epithelial surfaces alone or in collusion with other opportunistic pathogens such as
Staphylococcus aureus, the development of genetic resistance, and mechanisms to limit the entry of antimicrobials and/or increase their export through efflux pumps and/or enzymatically modify or destroy their antimicrobial properties (Nikaido, 2009; Blair et al., 2015; Vogwill et al., 2016). Studies have suggested that possible changes in bacterial metabolism that lead to reduced or no replication, may contribute to pathogen defense against antibiotic chemotherapy (Grant and Hung, 2013).

In addition to bacterial resistance strategies, it is possible that differences in the activities of antibiotics against intracellular bacteria may be due to relative accumulated concentrations within the cells (Van Bambeke et al., 2006; Poole, 2011).

In the current study, the three fluoroquinolone antibiotics CIP, LVX and OFX, were the most effective against extracellular 
and intracellular $P$. aeruginosa infection of hCFs, with CIP in particular showing the highest bactericidal activity. Killing of extracellular and intracellular $P$. aeruginosa by fluoroquinolone treatment has been observed also in primary human monocytes (Smith et al., 2000). The intracellular effects of different antibiotics have been tested also in Pseudomonas infected THP-1 cells: in this in vitro cell model, intracellular activity of antibiotics was dependent not only on the intrinsic growth inhibitory concentrations, but also on the maximal killing capacity of the antibiotic in a specific environment (Buyck et al., 2013). Unlike the fluoroquinolone antibiotics, the aminoglycoside GEN was able to kill extracellular but not intracellular $P$. aeruginosa; this may be due to the fact that aminoglycosides are poorly permeable, thus accumulating slowly within cells and only reaching active concentrations with long exposure times (Van Bambeke et al., 2006). Differences in Minimum Inhibitory Concentration (MIC) values for aminoglycosides have been recorded also during $P$. aeruginosa infection of THP-1 cells and were dependent on the cellular localization of the bacterium, with higher concentrations of antibiotics required to kill intracellularly localized organisms (Buyck et al., 2013). PMB could not sterilize $P$. aeruginosa-infected hCF monolayers completely: interestingly, this class of antibiotics has been reported to show efficacy against multidrug-resistant $P$. aeruginosa (MDR-P) (Poole, 2011), but has a high incidence of side effects, especially contact dermatitis, which limits its use in intensive antibiotic eye drop therapy (Jiaravuthisan and DeKoven, 2008; Poole, 2011). CXM treatment showed high antimicrobial activity against extracellular P. aeruginosa growth, but failed to clear intracellular infection. Limited intracellular penetration and accumulation within human cells has been described for this antibiotic, with intracellular CXM concentrations measured $\sim 100$ times lower than the concentration initially administrated (Darouiche and Hamill, 1994). In our model, treatment with the cellpermeant antibiotic CHL was totally ineffective in clearing both intracellular and extracellular bacteria. CHL is bacteriostatic for $P$. aeruginosa and would suppress bacterial replication if present in concentrations higher than the MIC. Since no inhibition of PAO1 growth was observed with CHL treatment, even at a concentration of $200 \mu \mathrm{g} / \mathrm{ml}$, it is likely that an effective MIC was not reached. In a retrospective review of therapeutic keratoplasty in Singapore, P. aeruginosa accounted for $58.7 \%$ of refractory microbial keratitis requiring penetrating keratoplasty. Interestingly, all patients were treated initially with gentamicin and a cephalosporin (Ti et al., 2007; Kaye et al., 2013).

In our hCF in vitro infection model, we observed eventual intracellular growth of $P$. aeruginosa after cessation of antibiotic treatment, followed by pathogen release and cell death. It has been reported that high levels of recurrent BK follow the cessation of antibiotic treatment (Kaye et al., 2013). This could be due to several factors other than antimicrobial resistance mechanisms, e.g., the early cessation of antibiotic treatment on apparent clearance of clinical infection and suboptimal levels of accumulated antibiotics within cells. Thus, bacteria residing within both corneal epithelial and fibroblast cells could be the triggers for observed recalcitrant infections. Relapse of Pseudomonas keratitis, in particular, occurs in human keratitis after apparent adequate antimicrobial chemotherapy (Van Horn et al., 1978) and the most recent data show that recurrent $P$. aeruginosa spp. are frequently isolated from repeat keratoplasties (Kaye et al., 2013). This indicates that $P$. aeruginosa spp. retention in corneal tissue is a relevant clinical problem. The pathogenesis of $P$. aeruginosa infection of corneal epithelial cells and stromal fibroblast cells share many similarities. Animal models have shown that retention of $P$. aeruginosa spp. in BK occurs within corneal epithelial cells (Lee et al., 2003a) and preliminary in vitro studies using electron microscopy, supports the view that a similar process can occur in corneal keratocytes (Elsahn et al., 2013). However, further animal and ideally human studies are required to assess whether this is the case in vivo.

A key consideration for antibiotic usage during $\mathrm{BK}$ is whether chemotherapy impacts on the inflammatory response induced by $P$. aeruginosa infection. A Cochrane review of studies of steroid use in microbial keratitis proposed the earlier use of anti-inflammatories to reduce damaging effects from the infective process (Herretes et al., 2014). Recently, we described the mechanisms whereby $P$. aeruginosa infection induces inflammasome-associated molecules in primary hCFs in vitro, which leads principally to the extracellular release of the cytokine IL-1 $\beta$ (Cendra et al., 2017). In our current study, the most effective anti-inflammatory antibiotics were CIP and GEN, which reduced IL-1 $\beta$ production from hCFs infected with high bacterial MOI $(=100)$. All the other antibiotics displayed some anti-inflammatory or nonstimulatory effects, but only with cells infected with lower MOI $(\leq 10)$. However, the increased levels of extracellular IL-1 $\beta$ quantified from infected monolayers treated with PMB and OFX suggests that these antibiotics are pro-inflammatory in our model. The variability in the hCF inflammatory response to some of the administered antibiotics during active infection in vitro is a novel finding and appears to be independent of compound activity against both extracellular and intracellular $P$. aeruginosa. The reported direct activation of the NLRP3 inflammasome-associated molecule by PMB could explain the increased IL-1 $\beta$ release from hCFs during treatment (Allam et al., 2011). The same hypothesis could extend to OFX treatment, and the possibility that both PMB and OFX treatment synergizes with bacterial infection to augment IL-1 $\beta$ production should not be excluded. However, whether these effects involve modulation of the activation of inflammasome-associated molecules is not known. The outcome of increased IL- $1 \beta$ in the presence of some of these antibiotics could be to enhance neutrophil influx (Smith et al., 1997), which could be viewed both as beneficial to the host for pathogen clearance, or potentially damaging as a consequence of eye tissue damage induced by the neutrophils.

Our study also highlighted that antibiotics varied in their cytotoxicity to primary hCF, which was more pronounced for OFX and PMB treatments. The pro-apoptotic effect of OFX could be due to stimulation of intracellular reactive oxygen species (ROS) production (Circu and Aw, 2010), which increases in a concentration- and time-dependent manner after OFX exposure, as observed in other cell-types such as chondrocytes 
(Sheng et al., 2013). Furthermore, the preservative benkalkonium chloride that was present in the OFX formulation used in this study, but absent in the fluoroquinolone CIP and LVX formulations, has been suggested to potentiate the OFX effect on ROS production and to activate the apoptotic receptor P2X7 (Dutot et al., 2006). In general, the combination of antibiotic and $P$. aeruginosa infection was not synergistic for cell cytotoxicity, and our study demonstrated that several of the antibiotics were able to reduce cytotoxicity, with CIP and LVX particularly effective treatments against high concentrations of bacterial infection.

In summary, our study identified the fluoroquinolone CIP, followed by LVX, as the most effective antibiotics for treating in vitro Pseudomonas infection of a monoculture model of primary hCFs. Both antibiotics eliminated extracellular and intracellular bacteria and reduced a cardinal inflammatory cytokine signal, which is observed in BK. The study also suggested that antibiotic concentration regimens must be sufficiently prolonged to eliminate $P$. aeruginosa intracellular infection, in order to minimize the risk of emergent bacteria that could initiate new cycles of infection.

\section{AUTHOR CONTRIBUTIONS}

MdMC, $\mathrm{PH}$, and MC designed the experimental research. MdMC performed the experiments. MdMC, $\mathrm{PH}$, and $\mathrm{MC}$ interpreted and analyzed the data. $\mathrm{MC}, \mathrm{MdMC}$, and $\mathrm{PH}$ wrote the manuscript. $\mathrm{MC}$ and $\mathrm{PH}$ contributed equally as PIs to this study.

\section{FUNDING}

MdMC was a postdoctoral research fellow supported by the Royal College of Surgeons of Edinburgh, Gift of Sight, National

\section{REFERENCES}

Ahmed, G. F., Elkhatib, W. F., and Noreddin, A. M. (2014). Inhibition of Pseudomonas aeruginosa PAO1 adhesion to and invasion of A549 lung epithelial cells by natural extracts. J. Infect. Public Health 7, 436-444. doi: 10.1016/j.jiph.2014.01.009

Allam, R., Darisipudi, M. N., Rupanagudi, K. V., Lichtnekert, J., Tschopp, J., and Anders, H. J. (2011). Cutting edge: cyclic polypeptide and aminoglycoside antibiotics trigger IL-1beta secretion by activating the NLRP3 inflammasome. J. Immunol. 186, 2714-2718. doi: 10.4049/jimmunol.1002657

Badenoch, P. R., and Coster, D. J. (1989). Selection of gentamicin-resistant variants of Pseudomonas aeruginosa in the rat cornea. J Ocul. Pharmacol. 5, 19-25. doi: 10.1089/jop.1989.5.19

Bargagna-Mohan, P., Lei, L., Thompson, A., Shaw, C., Kasahara, K., Inagaki, M., et al. (2015). Vimentin phosphorylation underlies myofibroblast sensitivity to withaferin a In Vitro and during corneal fibrosis. PLoS ONE 10:e0133399. doi: 10.1371/journal.pone.0133399

Blair, J. M., Webber, M. A., Baylay, A. J., Ogbolu, D. O., and Piddock, L. J. (2015). Molecular mechanisms of antibiotic resistance. Nat. Rev. Microbiol. 13, 42-51. doi: $10.1038 /$ nrmicro3380

Bleves, S., Viarre, V., Salacha, R., Michel, G. P., Filloux, A., and Voulhoux, R. (2010). Protein secretion systems in Pseudomonas aeruginosa: a wealth of pathogenic weapons. Int. J. Med. Microbiol. 300, 534-543. doi: 10.1016/j.ijmm. 2010.08.005
Eye Research Centre and the British Council for Prevention of Blindness. MdMC, MC, and $\mathrm{PH}$ acknowledge funding support from the Network for Anti-Microbial Resistance and Infection Prevention at the University of Southampton (NAMRIP, EPSRC reference EP/M027260/1).

\section{SUPPLEMENTARY MATERIAL}

The Supplementary Material for this article can be found online at: http://journal.frontiersin.org/article/10.3389/fmicb. 2017.01614/full\#supplementary-material

FIGURE S1 | PAO1 susceptibility to antibiotics during planktonic growth in LB medium. An initial PAO1 inoculum of $\sim 10^{5} \mathrm{CFU} / \mathrm{mL}, \sim 10^{6} \mathrm{CFU} / \mathrm{mL}$ and $\sim 10^{7}$ $\mathrm{CFU} / \mathrm{mL}$ was added to LB medium in presence of (A-G) CIP, LEV, PMB, GEN, OFX, CXM and $\mathrm{CHL}$ at 200, 100, 50, 10, 1, 0.1, and $0.01 \mu \mathrm{g} / \mathrm{mL}$ and optical density (OD) of the bacterial culture measured at $\lambda_{600} \mathrm{~nm}$ at $0,1,2,3,4,5,6,7,8$, 9 , and $24 \mathrm{~h}$ time-points. The symbols represent the mean and the error bars the standard error of the mean (SEM) from $n=3$ independent experiments.

FIGURE S2 | Planktonic growth of PAO1 in LB medium. The graph shows the OD at $\lambda_{600} \mathrm{~nm}$ of PAO1 from an initial inoculum of $\sim 10^{5} \mathrm{CFU} / \mathrm{mL}, \sim 10^{6} \mathrm{CFU} / \mathrm{mL}$ and $\sim 10^{7} \mathrm{CFU} / \mathrm{mL}$ over-time. The symbols represent the mean and the error bars the standard error of the mean (SEM) from $n=3$ independent experiments.

FIGURE S3 | Extracellular growth of PAO1 during hCF-infection. The graph shows the $\mathrm{CFU} / \mathrm{mL}$ enumerated extracellularly in infected monolayers at $\mathrm{MOI}=1\left(\sim 10^{5}\right.$ $\mathrm{CFU} / \mathrm{mL}), \mathrm{MOI}=10\left(\sim 10^{6} \mathrm{CFU} / \mathrm{mL}\right)$ and $\mathrm{MOI}=100\left(\sim 10^{7} \mathrm{CFU} / \mathrm{mL}\right)$ over-time. The symbols represent the mean and the error bars the SEM from $n=3$ independent experiments.

FIGURE S4 | hCF damage promoted by PAO1 infection and the antibiotics alone. hCF monolayers were infected with PAO1 at $\mathrm{MOI}=1,10$, and 100 (A) and treated with GEN, CXM, OFX, PMB, CIP, CHL and LVX at 50 and $200 \mu \mathrm{g} / \mathrm{mL}$ (B). $\mathrm{LDH}$ release was measured from supernatants after $0,1,3,6,9$, and $24 \mathrm{~h}$ of incubation of each condition and the percentage of cytotoxicity was calculated following the manufacturer's instructions. Non- infected and non-antibiotic treated monolayers were used as controls. The columns represent the mean percentage of cytotoxicity measured as $\mathrm{LDH}$ release from monolayers from $n=3$ independent experiments and the error bars represent the standard error of the means (SEM).

Borkar, D. S., Acharya, N. R., Leong, C., Lalitha, P., Srinivasan, M., Oldenburg, C. E., et al. (2014). Cytotoxic clinical isolates of Pseudomonas aeruginosa identified during the steroids for corneal ulcers trial show elevated resistance to fluoroquinolones. BMC Ophthalmol. 14:54. doi: 10.1186/1471-2415-14-54

Bucior, I., Tran, C., and Engel, J. (2014). Assessing Pseudomonas virulence using host cells. Methods Mol. Biol. 1149, 741-755. doi: 10.1007/978-1-4939-0473-0_57

Buyck, J. M., Tulkens, P. M., and Van Bambeke, F. (2013). Pharmacodynamic evaluation of the intracellular activity of antibiotics towards Pseudomonas aeruginosa PAO1 in a model of THP-1 human monocytes. Antimicrob. Agents Chemother. 57, 2310-2318. doi: 10.1128/AAC.02609-12

Carryn, S., Chanteux, H., Seral, C., Mingeot-Leclercq, M. P., Van Bambeke, F., and Tulkens, P. M. (2003). Intracellular pharmacodynamics of antibiotics. Infect. Dis. Clin. North Am. 17, 615-634. doi: 10.1016/S0891-5520(03)00066-7

Cendra, M. D. M., Christodoulides, M., and Hossain, P. (2017). Signaling mediated by toll-like receptor 5 sensing of Pseudomonas aeruginosa flagellin influences IL-1 $\beta$ and IL-18 production by primary fibroblasts derived from the human cornea. Front. Cell Infect. Microbiol. 7:130. doi: 10.3389/fcimb.2017.00130

Circu, M. L., and Aw, T. Y. (2010). Reactive oxygen species, cellular redox systems, and apoptosis. Free Radic. Biol. Med. 48, 749-762. doi: 10.1016/j.freeradbiomed. 2009.12.022

Cosma, C. L., Sherman, D. R., and Ramakrishnan, L. (2003). The secret lives of the pathogenic mycobacteria. Annu. Rev. Microbiol. 57, 641-676. doi: 10.1146/ annurev.micro.57.030502.091033 
Darouiche, R. O., and Hamill, R. J. (1994). Antibiotic penetration of and bactericidal activity within endothelial cells. Antimicrob. Agents Chemother. 38, 1059-1064. doi: 10.1128/AAC.38.5.1059

del Pozo, J. L., and Patel, R. (2007). The challenge of treating biofilm-associated bacterial infections. Clin. Pharmacol. Ther. 82, 204-209. doi: 10.1038/sj.clpt. 6100247

Donzis, P. B. (1998). Corneal ulcers from contact lenses during travel to remote areas. N. Engl. J. Med. 338, 1629-1630. doi: 10.1056/NEJM199805283382217

Dutot, M., Pouzaud, F., Larosche, I., Brignole-Baudouin, F., Warnet, J. M., and Rat, P. (2006). Fluoroquinolone eye drop-induced cytotoxicity: role of preservative in $\mathrm{P} 2 \mathrm{X} 7$ cell death receptor activation and apoptosis. Invest. Ophthalmol. Vis. Sci. 47, 2812-2819. doi: 10.1167/iovs.06-0224

Elsahn, A., Hossain, P., and Christodoulides, M. (2013). Microbiological and electron microscopic assessment of Pseudomonas aeruginosa infection of primary human corneal fibroblasts and epithelial cells in vitro. Invest. Ophthalmol. Vis. Sci. 54, 5218-5218.

Esen, M., Grassme, H., Riethmuller, J., Riehle, A., Fassbender, K., and Gulbins, E. (2001). Invasion of human epithelial cells by Pseudomonas aeruginosa involves src-like tyrosine kinases p60Src and p59Fyn. Infect. Immun. 69, 281-287. doi: 10.1128/IAI.69.1.281-287.2001

Flannagan, R. S., Heit, B., and Heinrichs, D. E. (2016). Intracellular replication of Staphylococcus aureus in mature phagolysosomes in macrophages precedes host cell death, and bacterial escape and dissemination. Cell Microbiol 18, 514-535. doi: $10.1111 / \mathrm{cmi} .12527$

Fleiszig, S. M., Zaidi, T. S., and Pier, G. B. (1995). Pseudomonas aeruginosa invasion of and multiplication within corneal epithelial cells in vitro. Infect. Immun. 63, 4072-4077.

Fleiszig, S. M., Zaidi, T. S., Preston, M. J., Grout, M., Evans, D. J., and Pier, G. B. (1996). Relationship between cytotoxicity and corneal epithelial cell invasion by clinical isolates of Pseudomonas aeruginosa. Infect. Immun. 64, 2288-2294.

Gellatly, S. L., and Hancock, R. E. (2013). Pseudomonas aeruginosa: new insights into pathogenesis and host defenses. Pathog. Dis. 67, 159-173. doi: 10.1111/ 2049-632X.12033

Gokhale, N. S. (2008). Medical management approach to infectious keratitis. Indian J. Ophthalmol. 56, 215-220. doi: 10.4103/0301-4738.40360

Grant, S. S., and Hung, D. T. (2013). Persistent bacterial infections, antibiotic tolerance, and the oxidative stress response. Virulence 4, 273-283. doi: 10.4161/ viru. 23987

Gresham, H. D., Lowrance, J. H., Caver, T. E., Wilson, B. S., Cheung, A. L., and Lindberg, F. P. (2000). Survival of Staphylococcus aureus inside neutrophils contributes to infection. J. Immunol. 164, 3713-3722. doi: 10.4049/jimmunol. 164.7.3713

Hammerschlag, M. R. (2002). The intracellular life of chlamydiae. Semin. Pediatr. Infect. Dis. 13, 239-248. doi: 10.1053/spid.2002.127201

Herretes, S., Wang, X., and Reyes, J. M. (2014). Topical corticosteroids as adjunctive therapy for bacterial keratitis. Cochrane Database Syst. Rev. 10, CD005430. doi: 10.1002/14651858.CD005430.pub3

Hung, M. C., Heckels, J. E., and Christodoulides, M. (2013). The adhesin complex protein (ACP) of Neisseria meningitidis is a new adhesin with vaccine potential. MBio 4:e00041-13. doi: 10.1128/mBio.00041-13

Jiaravuthisan, M. M., and DeKoven, J. G. (2008). Contact dermatitis to polymyxin B. Contact Derm. 59, 314-316. doi: 10.1111/j.1600-0536.2008.01428.x

Kaye, R., Kaye, A., Sueke, H., Neal, T., Winstanley, C., Horsburgh, M., et al. (2013). Recurrent bacterial keratitis. Invest. Ophthalmol. Vis. Sci. 54, 4136-4139. doi: 10.1167/iovs.13-12130

Kim, S. H., and Wei, C. I. (2007). Antibiotic resistance and Caco-2 cell invasion of Pseudomonas aeruginosa isolates from farm environments and retail products. Int. J. Food Microbiol. 115, 356-363. doi: 10.1016/j.ijfoodmicro.2006.12.033

Lebeaux, D., Ghigo, J. M., and Beloin, C. (2014). Biofilm-related infections: bridging the gap between clinical management and fundamental aspects of recalcitrance toward antibiotics. Microbiol. Mol. Biol. Rev. 78, 510-543. doi: 10.1128/MMBR.00013-14

Lee, E. J., Evans, D. J., and Fleiszig, S. M. (2003a). Role of Pseudomonas aeruginosa ExsA in penetration through corneal epithelium in a novel in vivo model. Invest. Ophthalmol. Vis. Sci. 44, 5220-5227.

Lee, E. J., Truong, T. N., Mendoza, M. N., and Fleiszig, S. M. (2003b). A comparison of invasive and cytotoxic Pseudomonas aeruginosa strain-induced corneal disease responses to therapeutics. Curr. Eye Res. 27, 289-299. doi: 10.1076/ceyr. 27.5.289.17220

Lovewell, R. R., Patankar, Y. R., and Berwin, B. (2014). Mechanisms of phagocytosis and host clearance of Pseudomonas aeruginosa. Am. J. Physiol. Lung. Cell Mol. Physiol. 306, L591-L603. doi: 10.1152/ajplung.00335.2013

Marquart, M. E. (2011). Animal models of bacterial keratitis. J. Biomed. Biotechnol. 2011:680642. doi: 10.1155/2011/680642

Masur, S. K., Idris, A., Michelson, K., Antohi, S., Zhu, L. X., and Weissberg, J. (1995). Integrin-dependent tyrosine phosphorylation in corneal fibroblasts. Invest. Ophthalmol. Vis. Sci. 36, 1837-1846.

Nikaido, H. (2009). Multidrug resistance in bacteria. Annu. Rev. Biochem. 78, 119-146. doi: 10.1146/annurev.biochem.78.082907.145923

O’Brien, T. P. (2003). Management of bacterial keratitis: beyond exorcism towards consideration of organism and host factors. Eye (Lond) 17, 957-974. doi: 10.1038/sj.eye.6700635

Okamoto, M., Takagi, M., Kutsuna, M., Hara, Y., Nishihara, M., Zhang, M. C., et al. (2004). High expression of interleukin-1beta in the corneal epithelium of MRL/lpr mice is under the control of their genetic background. Clin. Exp. Immunol. 136, 239-244. doi: 10.1111/j.1365-2249.2004.02428.x

Ong, H. S., and Corbett, M. C. (2015). Corneal infections in the 21st century. Postgrad. Med. J. 91, 565-571. doi: 10.1136/postgradmedj-2015-133323

Poole, K. (2011). Pseudomonas aeruginosa: resistance to the max. Front. Microbiol. 2:65. doi: $10.3389 /$ fmicb. 2011.00065

Ren, K., and Torres, R. (2009). Role of interleukin-1beta during pain and inflammation. Brain Res. Rev. 60, 57-64. doi: 10.1016/j.brainresrev.2008.12.020

Rybtke, M., Hultqvist, L. D., Givskov, M., and Tolker-Nielsen, T. (2015). Pseudomonas aeruginosa biofilm infections: community structure, antimicrobial tolerance and immune response. J. Mol. Biol. 427, 3628-3645. doi: 10.1016/j.jmb.2015.08.016

Shah, A., Sachdev, A., Coggon, D., and Hossain, P. (2011). Geographic variations in microbial keratitis: an analysis of the peer-reviewed literature. Br. J. Ophthalmol. 95, 762-767. doi: 10.1136/bjo.2009.169607

Shen, E. P., Hsieh, Y. T., Chu, H. S., Chang, S. C., and Hu, F. R. (2015). Correlation of Pseudomonas aeruginosa genotype with antibiotic susceptibility and clinical features of induced central keratitis. Invest. Ophthalmol. Vis. Sci. 56, 365-371. doi: $10.1167 /$ iovs.14-15241

Sheng, Z. G., Huang, W., Liu, Y. X., Yuan, Y., and Zhu, B. Z. (2013). Ofloxacin induces apoptosis via betal integrin-EGFR-Rac1-Nox2 pathway in microencapsulated chondrocytes. Toxicol. Appl. Pharmacol. 267, 74-87. doi: 10.1016/j.taap.2012.12.015

Smith, R. P., Baltch, A. L., Franke, M. A., Michelsen, P. B., and Bopp, L. H. (2000). Levofloxacin penetrates human monocytes and enhances intracellular killing of Staphylococcus aureus and Pseudomonas aeruginosa. J. Antimicrob. Chemother. 45, 483-488. doi: 10.1093/jac/45.4.483

Smith, R. S., Smith, T. J., Blieden, T. M., and Phipps, R. P. (1997). Fibroblasts as sentinel cells. Synthesis of chemokines and regulation of inflammation. Am. J. Pathol. 151, 317-322.

Stapleton, F., and Carnt, N. (2012). Contact lens-related microbial keratitis: how have epidemiology and genetics helped us with pathogenesis and prophylaxis. Eye (Lond) 26, 185-193. doi: 10.1038/eye.2011.288

Stapleton, W. M., Chaurasia, S. S., Medeiros, F. W., Mohan, R. R., Sinha, S., and Wilson, S. E. (2008). Topical interleukin-1 receptor antagonist inhibits inflammatory cell infiltration into the cornea. Exp. Eye Res. 86, 753-757. doi: 10.1016/j.exer.2008.02.001

Sy, A., Srinivasan, M., Mascarenhas, J., Lalitha, P., Rajaraman, R., Ravindran, M., et al. (2012). Pseudomonas aeruginosa keratitis: outcomes and response to corticosteroid treatment. Invest. Ophthalmol. Vis. Sci. 53, 267-272. doi: 10.1167/ iovs.11-7840

Taube, M. A., Del Mar Cendra, M., Elsahn, A., Christodoulides, M., and Hossain, P. (2015). Pattern recognition receptors in microbial keratitis. Eye (Lond) 29, 1399-1415. doi: 10.1038/eye.2015.118

Ti, S. E., Scott, J. A., Janardhanan, P., and Tan, D. T. (2007). Therapeutic keratoplasty for advanced suppurative keratitis. Am. J. Ophthalmol. 143, 755-762. doi: 10.1016/j.ajo.2007.01.015

Tuft, S., and Burton, M. (2013). The Royal College of Ophtalmologists- Focus. Microbial Keratitis, Autum. Available at: www.rcophth.ac.uk/core/core.picker/ download.asp?id=1826.2013 
Ulrich, M., Berger, J., Moller, J. G., and Doring, G. (2005). Moxifloxacin and ciprofloxacin protect human respiratory epithelial cells against Streptococcus pneumoniae, Staphylococcus aureus, Pseudomonas aeruginosa, and Haemophilus influenzae in vitro. Infection 33(Suppl. 2), 50-54. doi: 10.1007/s15010-005-8208-9

Van Bambeke, F., Barcia-Macay, M., Lemaire, S., and Tulkens, P. M. (2006). Cellular pharmacodynamics and pharmacokinetics of antibiotics: current views and perspectives. Curr. Opin. Drug Discov. Devel. 9, 218-230.

Van Horn, D. L., Davis, S. D., Hyndiuk, R. A., and Alpren, T. V. (1978). Pathogenesis of experimental Pseudomonas keratitis in the guinea pig: bacteriologic, clinical, and microscopic observations. Invest. Ophthalmol. Vis. Sci. 17, 1076-1086.

Vazirani, J., Wurity, S., and Ali, M. H. (2015). Multidrug-resistant Pseudomonas aeruginosa Keratitis: risk factors, clinical characteristics, and outcomes. Ophthalmology 122, 2110-2114. doi: 10.1016/j.ophtha.2015. 06.007

Vogwill, T., Comfort, A. C., Furio, V., and Maclean, R. C. (2016). Persistence and resistance as complementary bacterial adaptations to antibiotics. J. Evol. Biol. 29, 1223-1233. doi: 10.1111/jeb.12864

Wilson, J. W., Schurr, M. J., Leblanc, C. L., Ramamurthy, R., Buchanan, K. L., and Nickerson, C. A. (2002). Mechanisms of bacterial pathogenicity. Postgrad. Med. J. 78, 216-224. doi: 10.1136/pmj.78.918.216
Wong, Y., Sethu, C., Louafi, F., and Hossain, P. (2011). Lipopolysaccharide regulation of toll-like receptor-4 and matrix metalloprotease- 9 in human primary corneal fibroblasts. Invest. Ophthalmol. Vis. Sci. 52, 2796-2803. doi: 10.1167/iovs.10-5459

Yamamoto, N., Yamamoto, N., Petroll, M. W., Jester, J. V., and Cavanagh, H. D. (2006). Regulation of Pseudomonas aeruginosa internalization after contact lens wear in vivo and in serum-free culture by ocular surface cells. Invest. Ophthalmol. Vis. Sci. 47, 3430-3440. doi: 10.1167/iovs.05-1332

Young, D., Hussell, T., and Dougan, G. (2002). Chronic bacterial infections: living with unwanted guests. Nat. Immunol. 3, 1026-1032. doi: 10.1038/ni1102-1026

Conflict of Interest Statement: The authors declare that the research was conducted in the absence of any commercial or financial relationships that could be construed as a potential conflict of interest.

Copyright (c) 2017 del Mar Cendra, Christodoulides and Hossain. This is an openaccess article distributed under the terms of the Creative Commons Attribution License (CC BY). The use, distribution or reproduction in other forums is permitted, provided the original author(s) or licensor are credited and that the original publication in this journal is cited, in accordance with accepted academic practice. No use, distribution or reproduction is permitted which does not comply with these terms. 